\title{
Emerging Multifunctional Nanostructures
}

\author{
Hongyou Fan, ${ }^{1}$ Yunfeng Lu, ${ }^{2}$ Ganapathiraman Ramanath, ${ }^{3}$ and José A. Pomposo ${ }^{4}$ \\ ${ }^{1}$ Advanced Materials Laboratory, Sandia National Laboratories, Albuquerque, NM 87106, USA \\ ${ }^{2}$ Chemical and Biomolecular Engineering Department, University of California - Los Angeles (UCLA), CA 90095, USA \\ ${ }^{3}$ Materials Science \& Engineering Department, Rensselaer Polytechnic Institute, NY 12180, USA \\ ${ }^{4}$ New Materials Department, Centre for Electrochemical Technologies (CIDETEC), E20009 Donostia-San Sebastián, Spain
}

Correspondence should be addressed to José A. Pomposo, jpomposo@cidetec.es

Received 12 February 2009; Accepted 12 February 2009

Copyright ( 2009 Hongyou Fan et al. This is an open access article distributed under the Creative Commons Attribution License, which permits unrestricted use, distribution, and reproduction in any medium, provided the original work is properly cited.

The interest in emerging nanostructures is growing exponentially since they are promising building blocks for advanced multifunctional nanocomposites. In recent years, an evolution from the controlled synthesis of individual monodisperse nanoparticles to the tailored preparation of hybrid spherical and also unsymmetrical multiparticle nanostructures is clearly observed. As a matter of fact, the field of nanostructures built around a nanospecies such as inside, outside, and next to a nanoparticle is becoming a new evolving area of research and development with potential applications in improved drug delivery systems, innovative magnetic devices, biosensors, and highly efficient catalysts, among several others.

Emerging nanostructures with improved magnetic, conducting and "smart" characteristics are currently based on the design, synthesis, characterization and modeling of multifunctional nanoobject-based materials. In fact, coreshell nanoparticles and other related complex nanoarchitectures covering a broad spectrum of materials (from metal and metal oxide to fused carbon, synthetic polymer, and biopolymer structures) to nanostructure morphologies (spherical, cylindrical, star-like, etc.) are becoming the main building blocks for next generation of drug delivery systems, advanced sensors and biosensors, or improved nanocomposites. The five papers presented in this special issue examine the preparation and characterization of emerging multifunctional materials, covering from hybrid asymmetic structures to engineering nanocomposites.

In the first paper, the synthesis of nanometer-scale snowman-like asymmetric silica/polystyrene heterostructure with anisotropic functionality offering two-sided biological accessibility is reported. The morphology of the resulting asymmetric composite nanoparticles is illustrated by
TEM images. The interfacial behavior and amphiphilic characteristics of the hybrid nanoparticles as well as their functionalization with two different fluorescent molecules are demonstrated. This multifunctional materials will find important applications in biosensors, cell sorting, and fabrication of smart displays.

In the second paper, magnetic nanosized core-shell $\mathrm{Fe}_{3} \mathrm{O}_{4} / \mathrm{MnO}_{2}$ composite particles are synthesized by homogeneous precipitation with an $\mathrm{MnO}_{2}$ coating thickness of ca. $3 \mathrm{~nm}$ as demonstrated by TEM measurements. The hybrid nanoparticles exhibit super paramagnetic properties, and have better dispersivity than the starting materials and better ability of chemical adsorption. The potential use in dyestuff treatment is illustrated by methyl orange decoloration assays.

In the third paper, the confined arc plasma method is employed for the production of silver nanopowders with ultrafine and uniform particle size, high purity, welldispersed and quasispherical shape. The particle size, lattice parameter, microstructure, morphology, specific surface area, and pore parameters of the silver nanoparticles have been determined by a combination of techniques. This paper open the way to the synthesis of other emerging nanopowders by a convenient, inexpensive, and suitable method for mass production such as the confined arc plasma technique.

In the fourth paper, a simple method to fabricate a chemiresistor-type sensor based on dodecylamine-caped $\mathrm{Au}$ nanoparticles (average size 4-6nm) cross-linked with a phenylene ethynylene oligomer in a silica matrix is reported. This sensor minimizes the swelling transduction mechanism while optimizing the change in dielectric response. In fact, sensors prepared with this methodology show enhanced chemoselectivity for phosphonates which are useful surrogates for chemical weapons. 
In the final paper, engineering nanocomposites of polyamide 66 and Brazilian clay are investigated prepared via direct melt intercalation. XRD and TEM techniques are employed to investigate the interlayer spacing and the exfoliation degree in the nanocomposites, respectively. These nanostructured materials exhibit both interesting heat deflection temperatures and good thermal stability, both properties being interesting for industrial aplications.

Hongyou Fan Yunfeng Lu Ganapathiraman Ramanath

José A. Pomposo 

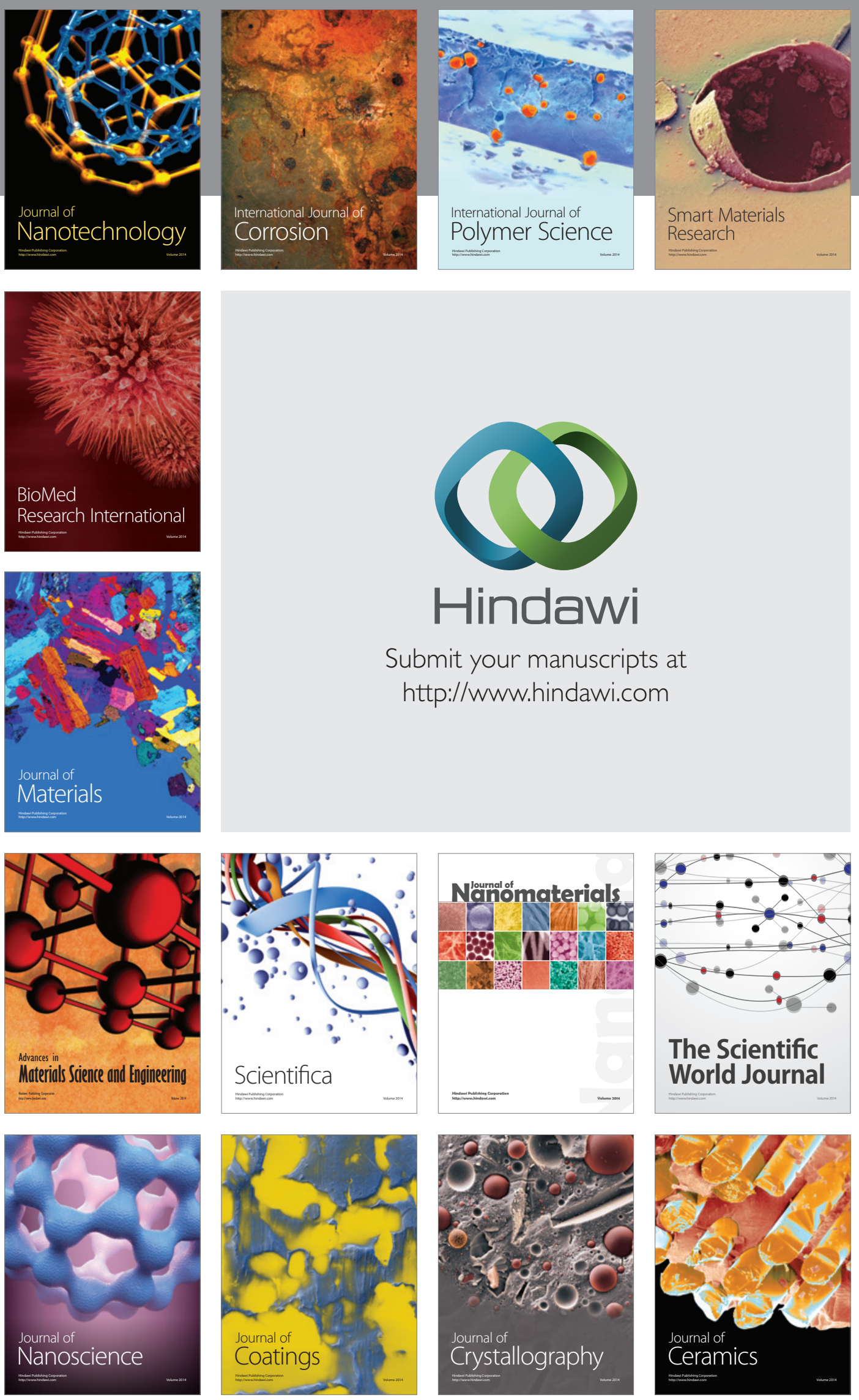

The Scientific World Journal

Submit your manuscripts at

http://www.hindawi.com

\section{World Journal}

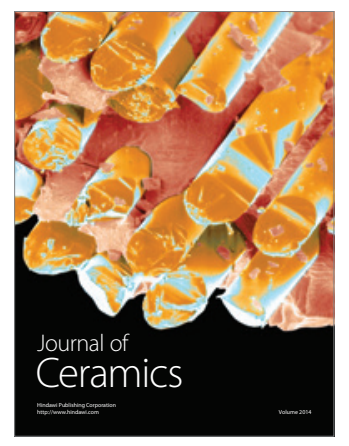

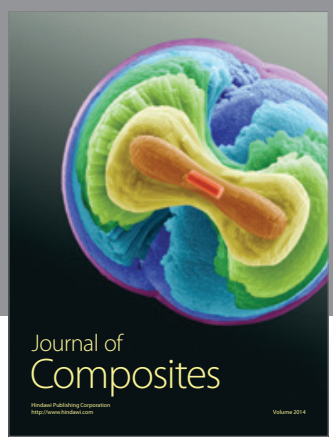
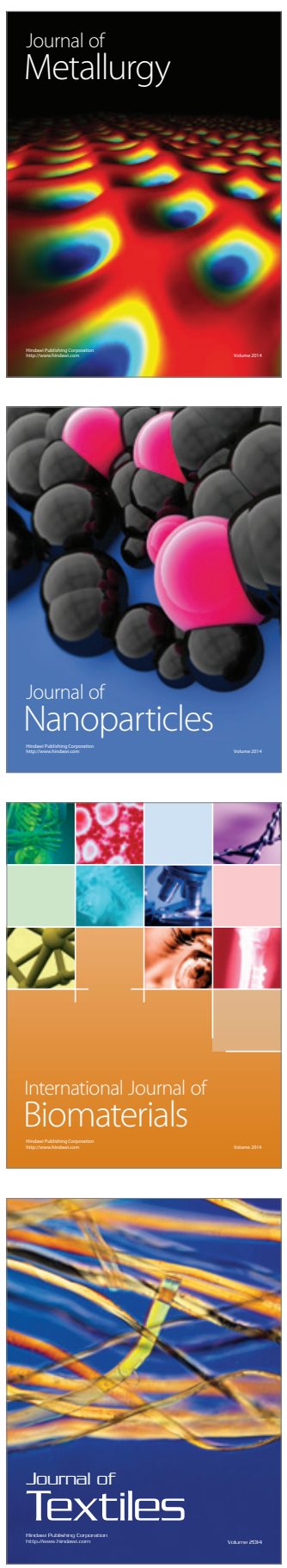